\title{
Profile of Ventilator Associated Pneumonia in Children Admitted to Pediatric Intensive Care Unit of a Tertiary Care Center in India
}

\author{
Asha PT ${ }^{1}$, Kulkarni R. ${ }^{2}$, Kinikar A. ${ }^{3}$, Rajput U. ${ }^{4}$, Valvi C. ${ }^{5}$, Dawre R. ${ }^{6}$ \\ ${ }^{1}$ Dr. Asha P.T., Senior Resident, ${ }^{2}$ Dr. Rajesh Kulkarni, Associate Professor, ${ }^{3}$ Dr. Aarti Kinikar, Professor and Head, \\ ${ }^{4}$ Dr. Uday Rajput, Associate Professor, ${ }^{5}$ Dr. Chhaya Valvi, Associate Professor, ${ }^{6}$ Dr. Rahul Dawre, Associate Professor, \\ All authors are affiliated with Dept. of Pediatrics, B.J. Govt. Medical College, Pune Maharashtra, India
}

Corresponding Author: Dr. Rajesh Kulkarni, Associate Professor. Department of Pediatrics, B.J. Govt. Medical College, Pune Maharashtra, India. E-mail: docrajesh75@yahoo.com

\begin{abstract}
Introduction: Limited literature is available on VAP in children, particularly from India.Hence this study aims to determine the incidence, clinical, laboratory, radiological and microbiological profile, risk factors, and outcomes of ventilator-associated pneumonia in pediatric patients. Methods: This is a prospective observational study which enrolled 125 children who were mechanically ventilated in the PICU of a tertiary teaching care hospital over a period of 2 years. Demographic, clinical, radiological and laboratory details were collected and CPIS was used to diagnose VAP. Endo tracheal aspirates were obtained at $48 \mathrm{hrs}$ and $96 \mathrm{hrs}$ of initiation of mechanical ventilation and were cultured as per standard guidelines. All the demographic, clinical, radiological and micro biological details were entered in SPSS version 17.0. Standard statistical tests were used to analyze data. Results: Among the 125 ventilated children, 44 were found to have VAP as per the CPIS showing its incidence of 35.2\%. In our study incidence of early VAP is $27.6 \%$ while that of late VAP is $75 \%$. The major risk factor for the incidence of VAP in this study was the duration of ventilation. VAP occurred in $75 \%$ of patients intubated for more than 5 days. Among 63 children who were reintubated, 34(53.9\%) developed VAP while the incidence of VAP was only $16.1 \%$ among those who were not reintubated (p value- 0.016 ). In this study out of the microorganisms isolated in patients with early VAP, $45 \%$ were Acinetobacter species, $20 \%$ Klebsiella pneumoniae and $10 \%$ Pseudomonas aeruginosa. Whereas in late onset VAP, Acinetobacter constituted $60 \%$ of all microbes while the rest comprised of Pseudomonas aeruginosa (20\%) and Klebsiella pneumonia (6.6\%). Conclusions: Significant risk factors in development of VAP were reintubation and duration of ventilation. The most common VAP pathogen was Acinetobacter species. Tracheostomized children have higher incidence of VAP but have higher recovery rates.
\end{abstract}

Key words: Ventilator associated pneumonia, Pediatric intensive care unit, Clinical pulmonary infection score, Multi drug resistance.

\section{Introduction}

Over the past fifty years, mechanical ventilation has undoubtedly represented an advance in the treatment of respiratory insufficiency. It can be lifesaving, but can lead to complications like pneumothorax, atelectasis, ventilator-associated pneumonia (VAP), obstruction of the tracheal tube during the intubation period, tracheal edema and tracheal stenosis after the extubation period [1].

Ventilator associated pneumonia (VAP) is defined as pneumonia which occurs after the patient has been on

Manuscript received: $10^{\text {th }}$ April 2019

Reviewed: $20^{\text {th }}$ April 2019

Author Corrected: $25^{\text {th }}$ April 2019

Accepted for Publication: $30^{\text {th }}$ April 2019 mechanical ventilation for more than 48 hours [1]. The prevalence ranges from $8 \%-28 \%$ in PICU [2,3]. VAP increases mortality and the length of ICU stay, which in turn increases the cost of treatment and chances of ventilator dependence.

The diagnosis of VAP is a critical issue but challenging with the current approach. With ambiguous clinical findings, diagnosis mainly depends on radiological and microbiological investigations [4]. Histopathology and culture of lung tissue remains the gold standard but, this is not feasible in most of the children and hence combinations of clinical, radiologic and microbiologic evidences are used. Early and accurate diagnosis is 


\section{Original Research Article}

essential to improve the outcomes, and to prevent overuse of antibiotics [5]. Compared to adult population with VAP, pediatric age group has distinctive characteristics with respect to susceptibility to infection and comorbid conditions, from the most extremely premature neonates to young infants, children, and adolescents and varying age-dependent tissue site antibiotic exposures [6,7]. With paucity of published literature in India in relation to incidence and microbiological profile of VAP this present study has been planned to address these aspects in children.

\section{Objective}

To determine the incidence, clinical, laboratory, radiological and microbiological profile, risk factors, and outcomes of ventilator-associated pneumonia in pediatric patients.

\section{Materials and Methods}

Setting: The present study was conducted between April 2014 to March 2016 in pediatric intensive care unit (PICU) of a tertiary care, referral and teaching hospital situated in an urban area with an average 1000 admissions per year.

Type of study: This is a prospective observational study which enrolled 125 children who were mechanically ventilated in the PICU.

Inclusion criteria: Inclusion criteria were children from 1 month to 12 years of age who required mechanical ventilation for more than 48 hours.

Exclusion criteria: Exclusion criteria was age less than 1 month and more than 12 years and readmission to PICU after shift out to ward.

Sample size: With an assumption of VAP incidence of $20 \%$ [2,3] a sample size for this study was calculated as 75 assuming incidence of VAP among pediatric patients in previous studies which was around $20 \%$, and permissible level of error in the estimated prevalence taken as $9 \%$.

Ethical consideration and Permission: The Institutional Ethics Community (IEC) approval was taken and patients were enrolled after written informed consent of parents.

Method: Pre-designed validated proforma captured the patient's demographic details, nutritional status and underlying primary diagnosis. Details about duration of hospital stay, ventilatory days, reintubation (patients who have received more than one endotracheal intubation and tracheostomy were documented. All enrolled children who were ventilated for more than 48 hours were monitored for features suggestive of nosocomial infection or VAP i.e. purulent tracheal secretions, new and persistent chest findings and body temperature instability. These children underwent partial septic screen including differential blood counts, blood cultures, tracheal aspirate for culture and sensitivity and chest X-ray.

Clinical pulmonary infection score (CPIS) was used for the diagnosis of VAP in (table 1) [8]. Data recorded were fever, leukocyte count, $\mathrm{PaO} 2 / \mathrm{FiO} 2$ (Partial arterial oxygen/Fraction of inspired oxygen), character of tracheal secretions and radiological findings. Chest radiographs were reported by a senior radiologist who was blinded to the clinical history of the patient.The nature of tracheal secretions was also noted by performing regular ETT suction.

Table-1: Clinical Pulmonary Infection Score.

\begin{tabular}{|c|c|c|c|}
\hline CPIS points & 0 & 1 & 2 \\
\hline Temperature $\left({ }^{0} \mathrm{C}\right)$ & $>36.5$ and $<38.4$ & 38.5 to 38.9 & $>39$ or $<36$ \\
\hline Leucocyte count(per mm3) & $4000-11,000$ & $<4000$, or $>11,000$ & $\begin{array}{c}<4,000 \text { or }>11,000+\text { band } \\
\text { forms }>500\end{array}$ \\
\hline PaO2/FiO2 ratio & $>240$ or ARDS & & $\begin{array}{c}<240 \text { and no evidence of } \\
\text { ARDS }\end{array}$ \\
\hline Chest X-ray infiltrates & No infiltrates & Diffuse & Localised \\
\hline Tracheal secretions & Rare & Abundant & Abundant+ purulent \\
\hline Microbiology & Negative & & Positive \\
\hline
\end{tabular}




\section{Original Research Article}

*The above parameters were routinely and meticulously recorded in patient charts at pre specified time points (at 48th hour and 96th hour following initiation of mechanical ventilation).

CPIS of more than 6 during first assessment and during second assessment were considered as having early VAP and late VAP respectively.

Endo tracheal aspirates were obtained at 48hours and 96hours of initiation of mechanical ventilation by a trained resident on duty with sterile mucous extractor under all aseptic precautions. The specimen collected was immediately transported aseptically to the laboratory within one hour of collection. Sample collected at night was stored at 4 degree centigrade overnight and sent to the laboratory next day morning for gram staining and culture. Purulent respiratory secretions were defined as secretions from the lungs, bronchi, or trachea that contain $>25$ neutrophils per low power field [8].

Culture and sensitivity of the aspirates were also done as per CLSI (Clinical and Laboratory Standard Institute) guidelines. Semi qualitative method was used to classify growth. Those with moderate to heavy growth were considered as culture positive. Multidrug-resistant pathogens were defined as those resistant to three or more antimicrobial classes. Clinical, radiological, and microbiological data of studied patients are summarized in table 2.

Data collected was tabulated in a Microsoft Excel 2010 spread sheet. All the demographic, clinical, radiological and micro biological details were entered in SPSS version 17.0. Descriptive statistics were used to calculate the frequencies of categorical data, and to compute means and standard deviations of continuous variables. Chi-square test and Fisher exact tests were used for the analysis of categorical variables (mode of ventilation, gender etc.). Student t-test was applied to find the difference between the means (SD) of continuous variables (length of stay, duration of ventilation etc.). A p-value of less than 0.05 was considered statistically significant.

\section{Results}

The total number of patents admitted to PICU during the study period was 1910 among which 246(12.8\%) children were ventilated. Out of this $125(50.8 \%)$ required mechanical ventilation for more than 48 hours. Among the 125 ventilated children, 44 were found to have VAP as per the CPIS showing its incidence of $35.2 \%$. In our study incidence of early VAP is $27.6 \%$ while that of late VAP is $75 \%$. Incidence of VAP was more in male children which was $25(56.8 \%)$. Amongst children diagnosed with VAP, 30 (68.1\%) were less than 5 years and $14(31.8 \%)$ were between 5 and 12 years of age.

Table-2: Clinical, radiological historical and microbiological data of studied patients.

\begin{tabular}{|c|c|c|c|}
\hline Factors & No VAP Group & VAP Group & p Value \\
\hline Gender & & & 0.161 \\
Male & $45(55.5)$ & $25(56.8)$ & $19(43.1)$ \\
Female & $36(44.5)$ & & $<0.001$ \\
\hline Duration of ventilation & $76(72.4)$ & $29(27.6)$ & $15(75)$ \\
\hline Less than 5 days & $5(25)$ & $34(77)$ & 0.016 \\
\hline More than 5 days & $29(35)$ & $4(9.1)$ & 0.451 \\
\hline Reintubation & $4(4.9)$ & $38(86.3)$ & 0.023 \\
\hline Blood culture & $61(75.3)$ & $30(68.1)$ & 0.016 \\
\hline Fever & $37(45.6)$ & $23(52.2)$ & 0.012 \\
\hline Leukocyte count & $17(20.9)$ & $22(50)$ & 0.007 \\
\hline PaO2/ FiO2 ratio & $9(11.1)$ & $31(70)$ & $<0.001$ \\
\hline Chest radiography (localised infiltrates) & $8(9.9)$ & $35(79.5)$ & $<0.001$ \\
\hline Tracheal secretions(purulent) & $6(7.5)$ & $3(75)$ & 0.125 \\
\hline Tracheal aspirate culture and sensitivity & $1(25)$ & $13(29.5)$ & 0.110 \\
\hline Tracheostomy & $33(40.7)$ & & \\
\hline Outcome (recovered) & & & \\
\hline
\end{tabular}




\section{Original Research Article}

Most common clinical condition requiring mechanical ventilation was respiratory failure $(60 \%)$ followed by altered mental status (24.8\%). The major risk factor for the incidence of VAP in this study was the duration of ventilation. VAP occurred in $75 \%$ of patients intubated for more than 5 days.

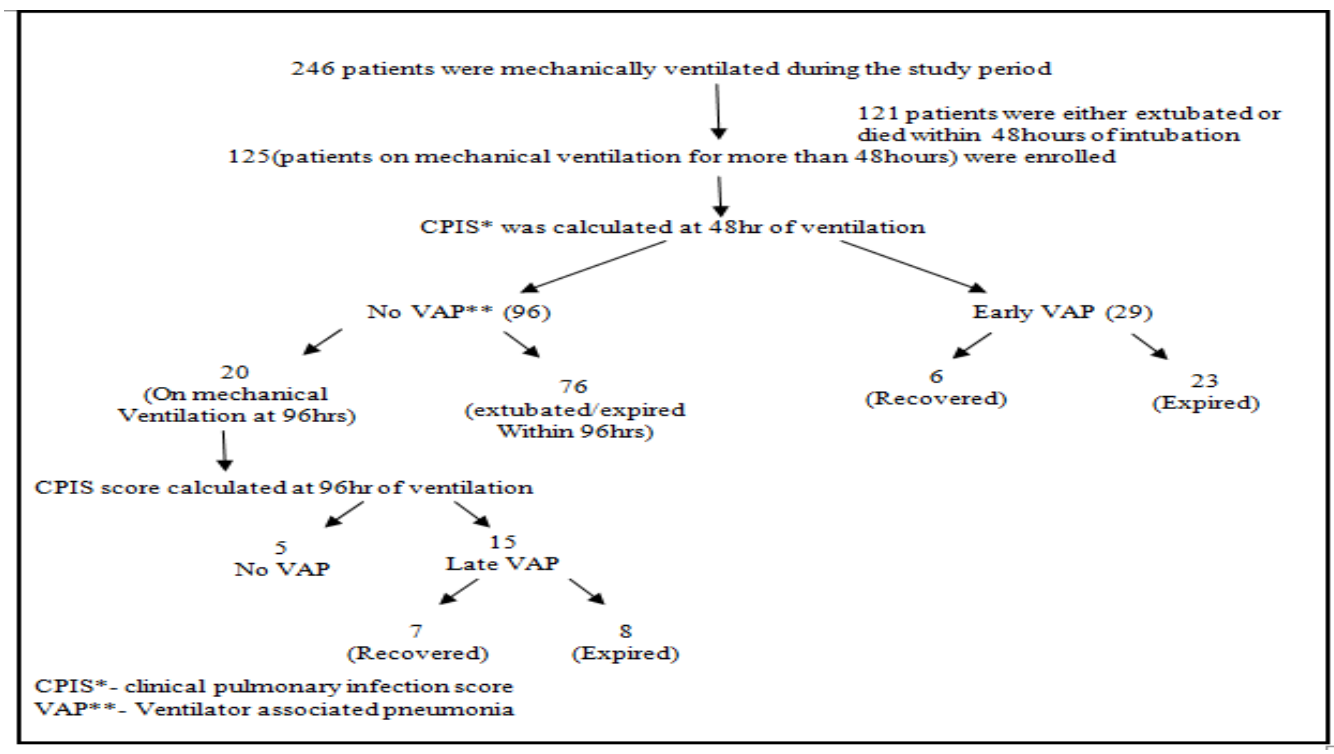

Figure-1: Study Flow Chart

Another risk factor was reintubation. Among 63 children who were reintubated, 34(53.9\%) developed VAP while the incidence of VAP was only $16.1 \%$ among those who were not reintubated ( $\mathrm{p}$ value- 0.016 ). Radiography (chest X-ray) revealed diffuse infiltrates in $69.1 \%$ among non VAP group, 55.2\% in early VAP and 33.3\% in late VAP group. We found that localized infiltrates were more specific with regard to VAP. Localized infiltrates were seen in only $11.1 \%$ among the non VAP group patients while it was $44.8 \%$ and $60.0 \%$ in the early VAP group and late VAP group respectively.

$65.5 \%$ of the patients with early VAP had purulent tracheal secretions while purulent secretions were found only in $40 \%$ of patients diagnosed with late VAP. In this study out of the microorganisms isolated in patients with early VAP, 45\% were Acinetobacter species, 20\% Klebsiella pneumoniae and 10\% Pseudomonas aeruginosa. Whereas in late onset VAP, Acinetobacter constituted $60 \%$ of all microbes while the rest comprised of Pseudomonas aeruginosa (20\%) and Klebsiella pneumonia (6.6\%).

$75 \%$ of patients who had undergone tracheostomy had VAP while incidence of VAP in patients who did not undergo tracheostomy was $33.8 \%$ ( $\mathrm{p}$ value $=0.125$ ). But in our study, it was seen that $100 \%$ of patients with VAP who had undergone tracheostomy recovered while recovery rate in other patients with VAP who had not undergone tracheostomy was only $24.3 \%$. So, the patients with VAP who had undergone tracheostomy had a better prognosis than those who did not undergo tracheostomy which was statistically significant ( $\mathrm{p}$ value $=0.004$ ).

It was observed that mortality in the patients with early VAP was $79.3 \%$ (23) while in those with late VAP was 53.3\% (8). Mortality rate in patients without VAP was $59.2 \%$ (48).

\section{Discussion}

The incidence of VAP in our study is $35.2 \%$ which is high compared to earlier studies conducted $[9,10]$. Being a tertiary government referral centre for many critical and end stage patients due to mainly economic reasons could have contributed to higher incidence. Incidence of VAP differs greatly based on setting and location in critically ill children in PICU.Although higher VAP rates have been reported before, our study reports high levels of MDR bacteria causing VAP $[11,12]$. Late referral, younger age, co-morbidities, and inadequate implementation of standard VAP Prevention guidelines may have contributed to higher VAP-related mortality in the present study. Highest incidence of VAP was seen in children between one and five years of age which has been reported by earlier studies on VAP. 


\section{Original Research Article}

Most common clinical condition requiring mechanical ventilation in this study was respiratory failure, similar findings were seen in other studies [13,14]. Most common risk factor for incidence of VAP which was also seen in other studies was increased duration of ventilation [15] and reintubation [16].

Also, it was observed that incidence of VAP was higher in those conditions which required prolonged ventilation like CNS diseases and tetanus while it was less in diseases which required lesser days on ventilator like shock and pneumonia. $100 \%$ of patients with early onset VAP and $93.3 \%$ with late onset VAP had new infiltrates ( $p$ value-0.007) on chest radiograph.Purulent secretions were seen only in $9.9 \%$ of patients without VAP.

The most common pathogen isolated from culture of tracheal aspirates in patients with VAP was Acientobacter species, and this was similar study conducted by Galal et al [17]. Majority of the isolates showed multibacterial resistance which is a concern in other studies also $[18,19]$.

Majority of the patients with tracheostomy (75\%) developed VAP. This was similar to the study conducted by Bigham [20]. Tracheostomy was found to be another independent risk factor as it is probable that leakage of pooled secretions around the tracheostomy tube into the trachea increases tracheal colonization and leads to VAP. Even though there was a higher incidence of VAP in tracheostomized patients, the recovery rate was significantly higher among them ( $p$ value $=0.110$ ). This has not been reported in other studies. We feel the recovery may be better in tracheostomized patients due to easier tracheobronchial toilet.

The strengths of our study include the prospective nature and adequate number of children enrolled.

Our study had a few limitations. Lung biopsy was not done in our study which may have limited the accuracy of the correlation with the risk factors and outcomes.

Invasive techniques to differentiate between infection and colonization such as Broncho Alveolar Lavage (BAL) were not available.Also we did not study association of VAP with some known risk factors like parenteral nutrition (as TPN facility is not available and aggressive enteral nutrition is advocated in our unit), acid suppression (not used routinely in ventilated children in our unit) and head end position( head elevation to 30 degree routinely done for all ventilated children in our PICU).

\section{Conclusion}

The incidence of VAP in this study was high when compared to other similar studies all over the world. Significant risk factors in development of VAP were reintubation and duration of ventilation.

Mortality rates were similar between patients with and without VAP. Even though there was a higher incidence of VAP in tracheostomized patients, the recovery rate was significantly higher among them.

The most common VAP pathogen was Acinetobacter species.

Gram negative bacteria (usually seen in hospital acquired infections) were isolated in most cases of early VAP which necessitates the need of early diagnosis of VAP for better treatment and outcome. Most of the pathogens exhibited multidrug resistance.

What This Study Adds: There is high incidence of VAP with MDR organisms. CPIS and tracheal aspirate cultures help in early diagnosis of VAP. Tracheostomized children have higher incidence of VAP but have higher recovery rates.

Contributors: APT-data collection, review of literature and drafting of manuscript, RKK-drafting of manuscript, review of literature, analysis, AAK. CTV, UR, RD, RB- drafting of manuscript, review of literature, analysis

Acknowledgement:We would like to thank department of Pathology, Microbiology and Radiology of BJGMC, Pune for their contributions to the study.

Funding: Nil, Conflict of interest: None initiated, Perission from IRB: Yes

\section{References}

1. Kendirli T, Kavaz A, Yalaki Z, et al. Mechanical ventilation in children. Turk J Pediatr. 2006 Oct-Dec;48 (4): 323-7.

2. Cooper VB, Haut C. Preventing ventilator-associated pneumonia in children: an evidence-based protocol. Crit Care Nurse. 2013 Jun;33(3):21-9; quiz 30. doi: 10.4037/ ccn 2013204.

3. Hamid MH, Malik MA, Masood J, et al. Ventilatorassociated pneumonia in children. J Coll Physicians Surg Pak. 2012 Mar;22(3):155-8. doi: 02.2012/JCPSP. 155158 . 


\section{Original Research Article}

4. Sachdev A, Chugh K, Sethi M, et al. Clinical Pulmonary Infection Score to diagnose ventilatorassociated pneumonia in children. Indian Pediatr. 2011 Dec;48 (12):949-54. Epub 2011 Mar 15.

5. Venkatachalam V, Hendley JO, Willson DF. The diagnostic dilemma of ventilator-associated pneumonia in critically ill children. PediatrCrit Care Med. 2011 May;12 (3): 286-96. doi:10. 1097/ PCC.0b013e3181 fe2ffb.

6. Lodha R, Kabra SK. Diagnosis of Ventilator Associated Pneumonia: Is There a Simple Solution? Indian Pediatrics 2011; 48:939-941

7. Joseph NM, Sistla S, Dutta TK, et al. Ventilatorassociated pneumonia in a tertiary care hospital in India: incidence and risk factors. J Infect Dev Ctries. 2009 Dec 15;3(10):771-7.

8. Grasso F, Chidini G, Napolitano L, Calderini E. Ventilator-associated pneumonia in children: evaluation of clinical pulmonary infection score in monitoring the course of illness. Crit Care. 2004;8(Suppl 1):P209. doi: $10.1186 / \mathrm{cc} 2676$

9. Gauvin F., Dassa C., Chaïbou M., Proulx F., Farrell C.A., Lacroix J. Ventilator-associated pneumonia in intubated children: Comparison of different diagnostic methods. Pediatr. Crit Care Med. 2003;7:437-443. doi: 10. 1097/01.PCC.0000090290.53959.F4.

10. Raymond J, Aujard Y. Nosocomial infections in pediatric patients: a European, multicenter prospective study. European Study Group. Infect Control Hosp Epidemiol. 2000 Apr; 21 (4):260-3.DOI:10.1086/50 1755

11. Patra PK, Jayashree M, Singhi S, et al. Nosocomial pneumonia in a pediatric intensive care unit. Indian Pediatr. 2007 Jul;44(7):511-8.

12. Sharma H, Singh D, Pooni P, Mohan U. A study profile of ventilator associated pneumonia in children in Punjab. J Trop Pediatr. 2009;55:393-5.
13. Silva DC, Shibata AR, Farias JA, et al. How is mechanical ventilation employed in a pediatric intensive care unit in Brazil? Clinics (Sao Paulo). 2009; 64 (12): 1161-6. doi: 10.1590/S1807-5932200900 1200005 .

14. Shirly GFA, Lakshmi S, Shanthi S, Darlington CD, Vinoth S. Clinical profile of children mechanically ventilated in a paediatric intensive care unit of a limited resource setting. Int J ContempPediatr2016;3:542-5.

15. Awasthi S, Tahazzul M, Ambast A, Govil YC, Jain A. Longer duration of mechanical ventilation was found to be associated with ventilator-associated pneumonia in children aged 1 month to 12 years in India. J Clin Epidemiol. 2013;66:62-6.

16. Bilan N, Habibi, P. Does Re-intubation Increased Risk of Ventilator-Associated Pneumonia (VAP) in Paediatric Intensive Care Unit Patients? Int J Pediatr. 2015; 3:411-5.

17. Galal YS, Youssef MR, Ibrahiem SK. VentilatorAssociated Pneumonia: Incidence, Risk Factors and Outcome in Paediatric Intensive Care Units at Cairo University Hospital. J Clin Diagn Res. 2016 Jun;10 (6): SC06-11. doi: 10.7860/JCDR/2016/ 18570. 7920. Epub 2016 Jun 1.

18. Vincent JL, Marshall JC, Namendys-Silva SA, et al. Assessment of the worldwide burden of critical illness: the intensive care over nations (ICON) audit. Lancet Respir Med. 2014 May;2(5):380-6. doi: 10.1016/S22132600(14)70061-X. Epub 2014 Apr 14.

19. Vincent JL, Rello J, Marshall J, et al. International study of the prevalence and outcomes of infection in intensive care units. JAMA. 2009 Dec 2;302(21):23239. doi: 10.1001/jama.2009.1754.

20. Bigham MT, Amato R, Bondurrant P, et al. Ventilator-associated pneumonia in the pediatric intensive care unit: characterizing the problem and implementing a sustainable solution. J Pediatr. 2009 Apr;154(4):582-587.e2. doi:10.1016/j.jpeds.2008. 10. 019. Epub 2008 Dec 3.

\section{How to cite this article?}

Asha PT, Kulkarni R, Kinikar A, Rajput U, Valvi C, Dawre R. Profile of Ventilator Associated Pneumonia in Children Admitted to Pediatric Intensive Care Unit of a Tertiary Care Center in India. Int J Pediatr Res. 2019;6(04):171176.doi:10.17511/ijpr.2019.i04.04 